\title{
MicroRNA-342-3p functions as a tumor suppressor by targeting LIM and SH3 protein 1 in oral squamous cell carcinoma
}

\author{
XIAOYAN SONG ${ }^{1 *}$, YONG JIN ${ }^{2 *}$, MINGYU YAN $^{3}$, YONGGANG ZHANG $^{4}$ and BING CHEN $^{5}$ \\ ${ }^{1}$ Department of Stomatology, Inner Mongolia Autonomous Region Maternal and Child Health Hospital, \\ Hohhot, Inner Mongolia 010020; ${ }^{2}$ Department of Stomatology, Tong-Liao City Hospital of Inner Mongolia, \\ Tong Liao, Inner Mongolia 028000; ${ }^{3}$ The Third Affiliated Hospital, Inner Mongolia Medical University, Baotou, \\ Inner Mongolia 014010; ${ }^{4}$ Department of General Surgery, Affiliated Renmin Hospital of Inner Mongolia Medical \\ University, Huhhot, Inner Mongolia 010017; ${ }^{5}$ Department of General Surgery, Inner Mongolia Autonomous \\ Region Maternal and Child Health Hospital, Huhhot, Inner Mongolia 010020, P.R. China
}

Received February 13, 2018; Accepted August 22, 2018

DOI: $10.3892 / 01.2018 .9637$

\begin{abstract}
Although microRNA-342-3p (miR-342-3p) deregulation has been implicated in the development of a variety of cancer types, its role in oral squamous cell carcinoma (OSCC) progression remains unclear. Overexpression of LIM and SH3 protein 1 (LASP1) in OSCC tissues, and its promotion of OSCC cell proliferation were recently reported. However, the regulatory mechanism underlining LASP1 expression remains unknown. In the present study, the notable downregulation of miR-342-3p in OSCC cell lines and clinical specimens was revealed. The Cell Counting kit-8 and 5-bromo-2-deoxyuridine-incorporation assays demonstrated that miR-342-3p suppressed OSCC cell proliferation. Additionally, LASP1 was identified as a target gene of miR-342-3p through bioinformatics analysis and luciferase reporter assays. Further experiments suggested that overexpression of LASP1 attenuated the suppressive effect of miR-342-3p on the proliferation of OSCC cells. In conclusion, the present data suggest that miR-342-3p functions as a tumor suppressor in OSCC via targeting of LASP1 and may be a promising therapeutic target for OSCC.
\end{abstract}

Correspondence to: Dr Bing Chen, Department of General Surgery, Inner Mongolia Autonomous Region Maternal and Child Health Hospital, 6 Gongyuandong Road, Huhhot, Inner Mongolia 010020, P.R. China

E-mail: mancbsxy@163.com

${ }^{*}$ Contributed equally

Abbreviations: miR-342-3p, microRNA-342-3p; LASP1, LIM and SH3 protein 1; OSCC, oral squamous cell carcinoma; 3'-UTR, 3'-untranslated region

Key words: microRNA-342-3p, oral squamous cell carcinoma, LIM and $\mathrm{SH} 3$ protein 1

\section{Introduction}

Oral squamous cell carcinoma (OSCC), the most common malignancy of the head and neck, accounts for $2 \%$ of cancer-related mortalities worldwide, with a steadily increasing incidence (1). Despite advances in treatment strategies, which have included a combination of surgery, radiotherapy and chemotherapy, the survival rates of patients with OSCC remain at $<50 \%$ (2). The deregulated proliferation and apoptosis of cancer cells have been reported to serve a critical role in the initiation and progression of OSCC (3). Therefore, an improved understanding of the molecular basis underlying this malignancy could facilitate the development of an effective therapeutic strategy.

MicroRNAs (miRNAs/miRs), a type of non-coding RNA, are capable of binding the 3'-untranslated region (3'-UTR) of the target mRNA and repressing gene expression by either enhancing mRNA degradation or suppressing mRNA translation (4). Due to their ability to modulate gene expression, miRNAs have been reported to participate in a wide range of cellular processes, including cell proliferation, senescence, apoptosis and migration (5). Accumulating evidence has demonstrated that the deregulation of miRNAs mediates the development of numerous malignancies, including cancer of the breast, gastric system, prostate and large intestine, through the modulation of tumor cell viability, angiogenesis and metastasis (6). In human OSCC, miRNAs can serve either an oncogenic or tumor-suppressing role during tumorigenesis. For example, certain miRNAs, including miR-21, miR-222 and miR-448, were discovered to be overexpressed in OSCC and function as oncogenes (7-9). By contrast, other miRNAs, including miR-9, miR-99a and miR-491-5p, were found to be downregulated in OSCC and exhibit tumor-suppressive behavior (10-13).

miR-342-3p serves a critical role in numerous physiological and pathological processes. miR-342-3p negatively regulates cell viability by repressing the anti-apoptotic gene network in human and mouse macrophages (14). Furthermore, it was identified as a powerful enhancer of adipogenesis by targeting C-terminal-binding protein 2 (CtBP2), leading to the release 
of the key adipogenic regulator CCAAT-enhancer-binding protein $\alpha$ from CtBP2 binding (15). In addition, miR-342-3p serves a role in the osteogenic differentiation of umbilical cord mesenchymal stem cells (16). Recently, evidence suggested that miR-342-3p is abnormally expressed in tissues of various types of cancer and participates in tumorigenesis. Gao et al (17) reported that miR-342-3p exhibited decreased expression in hepatocellular carcinoma and that it may be used as an independent predictor for poor prognoses. In non-small cell lung cancer (NSCLC), miR-342-3p demonstrated decreased expression and was shown to serve an inhibitory role in cell proliferation by targeting anterior gradient protein 2 (18). miR-342-3p was also reported to be downregulated in cervical cancer tissue and repressed cell proliferation by targeting forkhead box protein M1, a well-established oncogenic factor (19). Although these studies demonstrate the important role of miR-342-3p in cancer progression, its expression in OSCC tissues and its function in OSCC progression remain unclear.

In the present study, the expression of miR-342-3p were detected OSCC cells and tissues using reverse transcription-quantitative PCR. The effect of miR-342-3p overexpression or silencing on the proliferation of OSCC cells was explored using Cell Counting Kit-8 (CCK-8), colony formation assay and 5-Bromo-2-deoxyuridine (BrdU)-incorporation assay. Finally, luciferase assays, western blot analysis and rescue experiments were performed to investigate whether LIM and SH3 protein 1 (LASP1) was the functional mediator of miR-342-3p.

\section{Materials and methods}

Cell lines and reagents. Human OSCC lines, including OC3, SCC-4, Tca8113, SCC-9 and OEC-M1, and human normal oral keratinocytes (hNOKs) were obtained from the State Key Laboratory of Oral Diseases, Sichuan University (Sichuan, China) and the State Key Laboratory of Oncology in South China, Sun Yat-Sen University (Guangdong, China), respectively. The primary antibody to LASP1 was purchased from Sigma-Aldrich (SAB2101318); Merck KGaA (Darmstadt, Germany) and $\alpha$-tubulin antibody (sc-398103) was obtained from Santa Cruz Biotechnology, Inc. (Dallas, TX, USA).

Cell culture. Human OSCC lines, including OC3, SCC-4, Tca8113, SCC-9 and OEC-M1, and human normal oral keratinocytes (hNOKs) were cultured in Dulbecco's modified Eagle's medium (DMEM) (Invitrogen; Thermo Fisher Scientific, Inc., Waltham, MA, USA) supplemented with $10 \%$ FBS (Gibco; Thermo Fisher Scientific, Inc.), 10 mM HEPES, $1 \mathrm{mM}$ sodium pyruvate, $2 \mathrm{mM}$ glutamine, $100 \mathrm{U} / \mathrm{ml}$ penicillin and $100 \mathrm{mg} / \mathrm{ml}$ streptomycin at $37^{\circ} \mathrm{C}$ in a humidified incubator with $5 \% \mathrm{CO}_{2}$. The cells were passaged every 2 or 3 days. The cells at passage 10-15 were used in this study.

Tissue samples. The present study was approved by the Ethics Committee of The Third Affiliated Hospital, Inner Mongolia Medical University (Inner Mongolia, China). In total, 30 paired OSCC tumor tissues and the adjacent non-cancerous specimens were collected from patients undergoing surgical resection at The Third Affiliated Hospital, Inner Mongolia Medical University. No patient had received any therapy, including radiotherapy or chemotherapy, prior to surgery. Patients provided written informed consent prior to study initiation. All tissue samples were frozen in liquid nitrogen once the diagnosis had been confirmed by tissue pathology.

Reverse transcription-quantitative PCR (RT-qPCR). miRNA was extracted from human tissue samples and cultured cells using the mirVana miRNA Isolation kit (Ambion; Thermo Fisher Scientific, Inc.), following the manufacturer's protocol. Expression of miR-342-3p was detected on a CFX96 Touch $^{\mathrm{TM}}$ Real-Time PCR Detection system (Bio-Rad Laboratories, Inc., Hercules, CA, USA) using the PrimeScript miRNA RT-PCR kit (Takara Biotechnology Co., Ltd., Dalian, China), according to the manufacturer's protocols, and U6 was used to normalize miRNA levels. The thermocycling conditions of quantitative PCR were as follows: $94^{\circ} \mathrm{C}$ for $45 \mathrm{sec}, 59^{\circ} \mathrm{C}$ for $45 \mathrm{sec}$ and $72^{\circ} \mathrm{C}$ for $60 \mathrm{sec}$, for 35 cycles and $72^{\circ} \mathrm{C}$ for $10 \mathrm{~min}$. The sequences of the primers used were as follows: miR-342-3p forward, 5'-TCCTCGCTCTCACACAGAAATC-3' and reverse, 5'-TAT GGTTGTTCACGACTCCTTCAC-3'; and U6 forward, 5'-ATT GGAACGATACAGAGAAGATT-3' and reverse, 5'-GGA ACGCTTCACGAATTTG-3'.

Total RNA was isolated from cells using TRIzol (Invitrogen; Thermo Fisher Scientific, Inc.) and reverse-transcribed into cDNA using the PrimeScript RT reagent kit (Takara Biotechnology Co.) with the following temperature protocol; $25^{\circ} \mathrm{C}$ for $5 \mathrm{~min}$, followed by $42^{\circ} \mathrm{C}$ for $60 \mathrm{~min}$ and $70^{\circ} \mathrm{C}$ for 5 min. The RT-qPCR was performed on a CFX96 Touch $^{\mathrm{TM}}$ Real-Time PCR Detection system (Bio-Rad Laboratories, Inc.) using the Power SYBR Green Master mix (Applied Biosystems; Thermo Fisher Scientific, Inc.) and GAPDH was used to normalize mRNA levels. The relative expression levels of miRNA and mRNA were calculated using $2^{-\Delta \Delta C q}$ method (20). The primer sequences were as follows: LASP1 forward, 5'-GGTGCGGCAAGATCGTGTA-3' and reverse, 5'-GGTCTCGCAATGGAA-3'; and GAPDH forward, 5'-ACA ACTTTGGTATCGTGGAAGG-3' and reverse, 5'-GCCATC ACGCCACAGTTTC-3'.

Transient transfection of miR-342-3p mimics or inhibitors. Given that Tca8113 and SCC-9 cell lines express an intermediate level of miR-342-3p compared with other OSCC cell lines (OC3, SCC-4 and OEC-M1), they were selected for subsequent gain-of-function and loss-of-function experiments. miR-342-3p mimics (100 nmol/l) or antagomiR-342-3p (100 nmol/l) (Guangzhou RiboBio Co., Ltd., Guangzhou, China) were transfected into Tca8113 or SCC-9 cells with Lipofectamine ${ }^{\circledR}$ RNAiMAX Transfection Reagent (Invitrogen; Thermo Fisher Scientific, Inc.), following the manufacturer's protocols, and used for subsequent experiments at 24 or $48 \mathrm{~h}$ post-transfection. miR-342-3p mimic sense sequence was, 5'UCUCACACAGAAAUCGCACCCGU-3'; The antagomir sequence was, 5'ACGGGTGCGATTTCTGTGTGAGA-3'.

Cell proliferation assays. The cells were seeded in 96-well plates at 3,000 cells/well. After $72 \mathrm{~h}$ of culture in DMEM supplemented with $10 \% \mathrm{FBS}$ at $37^{\circ} \mathrm{C}$, cell proliferation was measured with Cell Counting kit-8 (CCK-8) (Dojindo Molecular Technologies, Inc., Kumamoto, Japan) following the manufacturer's protocols. Assays were performed in 
triplicate and the results are presented as the mean \pm standard deviation (SD).

Colony formation assay. To evaluate the colony formation ability of tumor cells, the cells were seeded at 2,000 cells/well in a 6-well plate. Cells were cultured in DMEM supplemented with $10 \% \mathrm{FBS}$ at $37^{\circ} \mathrm{C}$ for 2 weeks and then fixed with fixed with pure methanol for $15 \mathrm{~min}$ at room temperature. Subsequent to being stained with $0.5 \%$ crystal violet solution for $20 \mathrm{~min}$, the colonies were counted using ImageJ software version 2.0.0 (National Institutes of Health, Bethesda, MD, USA). Assays were performed in triplicate and the results are presented as the mean \pm SD.

5-Bromo-2-deoxyuridine (BrdU)-incorporation assay. A total of $5 \times 10^{4} \mathrm{Tca} 8113$ or SCC-9 cells were incubated with $10 \mu \mathrm{M}$ BrdU for $30 \mathrm{~min}$ at $37^{\circ} \mathrm{C}$ in a humidified incubator with $5 \% \mathrm{CO}_{2}$. Following a wash with PBS, the cells were rinsed thoroughly and incubated with $6 \mathrm{M} \mathrm{HCl}$ in PBS at room temperature for $30 \mathrm{~min}$. Subsequent to two washes with $0.1 \mathrm{M}$ borate buffer and a wash with PBS containing $0.1 \%$ bovine serum albumin (BSA, Sigma-Aldrich, Merck KGaA, Darmstadt, Germany), the cells were incubated with $10 \mu \mathrm{g} / \mathrm{ml}$ anti-BrdU fluorescein (BD Biosciences, San Jose, CA, USA) for $1 \mathrm{~h}$ at room temperature in the dark, followed by flow cytometry analysis in the FACSCanto $^{\text {TM }}$ II cell analyzer (BD Biosciences, San Jose, CA, USA). Data were analyzed using FlowJo v.10 software (FlowJo LLC, Ashland, OR, USA).

Luciferase reporter assay. Both TargetScan (www.targetscan. org) and miRanda (http://diana.imis.athena-innovation. gr/DianaTools/index.php) predicted LASP1 as a potential target gene of miR-342-3p with binding site located at 3'UTR of LASP1. Therefore, a duel luciferase reporter assay was used to examine whether miR-342-3p binds to the 3'UTR of LASP1 mRNA. First, the plasmid with LASP1 3'UTR oligonucleotide fragment was constructed and inserted in (pGL3-LASP1-WT). Then, we mutated miR-342-3p binding sequences at LASP1 3'UTR (pGL3-LASP1-M) from 5'-...GUGUGAG...-3' to 5'-... CACACUC...-3'. The effectiveness of constructs was verified by sequencing. Tca8113 or SCC-9 cells were seeded into 24-well plates $\left(5 \times 10^{4}\right.$ cell/well). The following day, $5 \mathrm{ng} \mathrm{pRL}-\mathrm{TK}$ vector (an internal control) and $50 \mathrm{ng}$ pGL3 luciferase reporter plasmid (Promega Corporation, Madison, WI, USA) carrying the wild-type (WT) or mutant LASP1 3'-UTR were co-transfected into Tca8113 or SCC-9 cells along with miR-342-3p mimics or antagomiR-342-3p using the Lipofectamine 2000 transfection reagent (Invitrogen; Thermo Fisher Scientific, Inc.). At $48 \mathrm{~h}$ post-transfection, the cells were harvested for luciferase activity measurement using the Dual-Luciferase Reporter Assay system (Promega Corporation, Madison, WI, USA) following the manufacturer's protocol. The Renilla luciferase activity was used as an internal control and the firefly luciferase activity was calculated as the mean \pm SD after being normalized by Renilla luciferase activity.

Western blotting. The cells were lysed using RIPA buffer (Beyotime Institute of Biotechnology, Shanghai, China) containing a protease inhibitor cocktail (Roche Diagnostics $\mathrm{GmbH}$, Mannheim, Germany). After $40 \mathrm{~min}$, the cells were centrifuged for $20 \mathrm{~min}$ at $4^{\circ} \mathrm{C}$ at $12,000 \mathrm{x}$ g speed, and then the supernatant was carefully removed to obtain the total protein. The protein concentration was measured using the Pierce BCA Protein Assay kit (Thermo Fisher Scientific, Inc.) following the manufacturer's protocols. The detailed protocol has been described previously (21). Equal amounts of protein per lane $(50 \mu \mathrm{g})$ were separated by $10 \%$ SDS-PAGE gel and transferred to a nitrocellulose membrane (Merck KGaA). Subsequent to being blocked with $5 \%$ non-fat milk in tris-buffered saline/Tween-20 (TBST) for $1 \mathrm{~h}$ at room temperature, the membranes were incubated with the aforementioned primary antibodies (Rabbit anti-LASP1 antibody, 1:500; Mouse anti- $\alpha$-tubulin antibody, $1: 2,000)$ at $4^{\circ} \mathrm{C}$ overnight. Following three washes with TBST, the membranes were incubated with horseradish peroxidase-conjugated goat anti-mouse IgG (1:2,000, cat. no. KC-MM-035; Kangcheng Bio-tech, Shanghai, China) and horseradish peroxidase-conjugated goat anti-rabbit IgG (1:2,000, cat. no.: KC-RB-035; Kangcheng) for $1 \mathrm{~h}$ at room temperature and washed again 3 times in TBST. The immunocomplexes were detected using by an ECL ${ }^{\mathrm{TM}}$ Western Blotting Detection Reagents (GE Healthcare, Chicago, IL, USA).

Plasmid and siRNA transfection. The Gateway Technology (Invitrogen; Thermo Fisher Scientific, Inc.) was used in this study to clone LASP1 gene into a pcDNA3.1 plasmid (Invitrogen; Thermo Fisher Scientific, Inc.). pcDNA3.1-LASP1 were transfected into the cells with Lipofectamine RNAiMAX Transfection Reagent (Invitrogen; Thermo Fisher Scientific, Inc.). The siRNA oligos targeting Lasp1 (5'-CUUAUCCAG ACAGUUCACCdTdT-3';) and the negative control siRNA (siCtrl) (5'-AGAGAUGUAGUCGCUCGCUdTdT-3';) were synthesized by Zoonbio Biotechnology Co., Ltd (Nanjing, China). A total of $20 \mathrm{nM}$ siRNA oligos were transfected into the cells with Lipofectamine RNAiMAX Transfection Reagent. After $12 \mathrm{~h}$ of transfection, the cells underwent subsequent experimentation.

Statistical analysis. Each experiment was performed in triplicate. The data are presented as the mean \pm SD. Statistical analysis was performed using the SPSS v17.0 software (SPSS, Inc., Chicago, IL, USA). The unpaired Student's t-test was used for comparing the statistical significance between 2 groups. The statistical differences for comparing $>2$ groups were analyzed using the one-way analysis of variance followed by Dunnett's post hoc test. Pearson's correlation analysis was performed to examine the correlation between the miR-342-3p and LASP1 levels in clinical specimens. $\mathrm{P}<0.05$ was considered to indicate a statistically significant difference.

\section{Results}

OSCC cells and tissues exhibit reduced miR-342-3p expression. To explore the role of miR-342-3p in OSCC, the expression profile of miR-342-3p in hNOKs and 5 oral squamous cell carcinoma cell lines (OC3, SCC-4, Tca8113, SCC-9 and OEC-M1) was examined first. Compared with that in hNOK cells, miR-342-3p expression was significantly decreased in all 5 OSCC cell lines (Fig. 1A). The expression levels of miR-342-3p in 30 pairs of OSCC tumor tissues and 

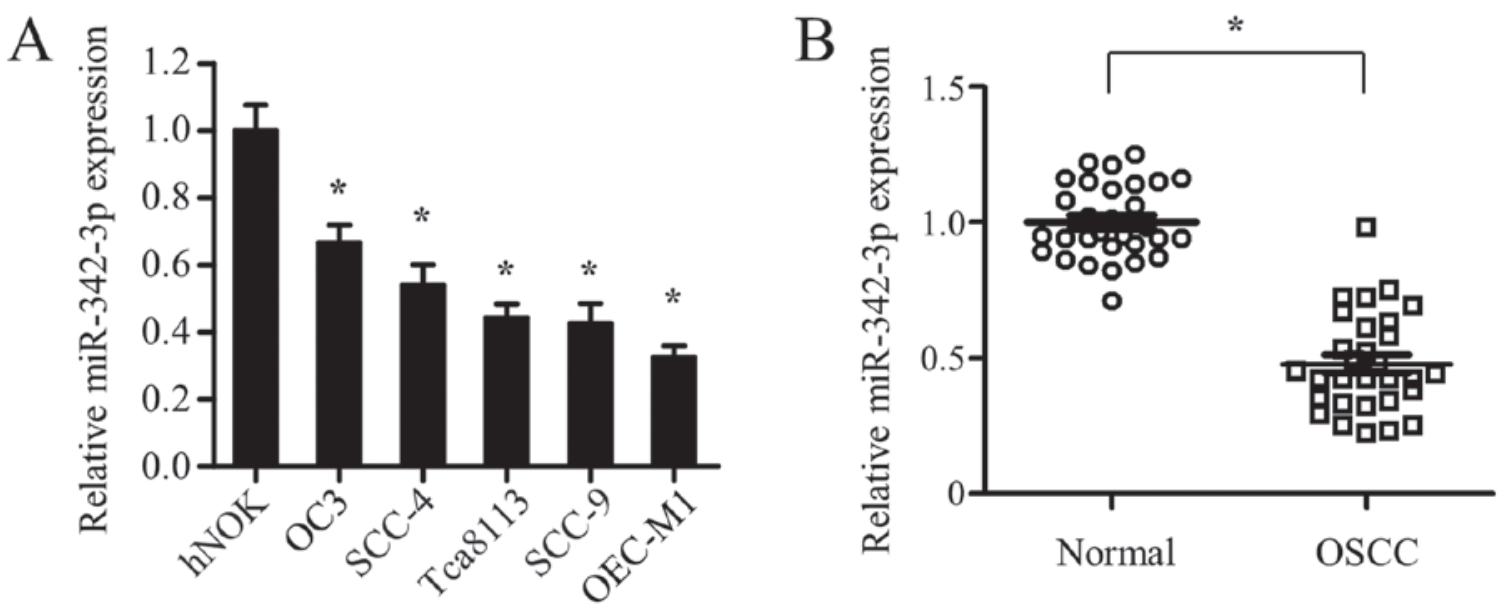

Figure 1. Reduced expression of miR-342-3p in OSCC cells and tissues. (A) qPCR analysis of miR-342-3p expression in hNOKs and 5 OSCC cell lines (OC3, SCC-4, Tca8113, SCC-9 and OEC-M1). (B) Comparison of miR-342-3p expression levels in OSCC tumor tissues and the adjacent non-tumorous tissues through qPCR analysis $(\mathrm{n}=30)$. U6 was used as the endogenous control. "P<0.05 vs. OSCC tumor tissues. miR, microRNA; qPCR, quantitative polymerase chain reaction; hNOK, human normal oral keratinocyte; OSCC, oral squamous cell carcinoma.

adjacent non-tumorous tissues were analyzed next. Consistent with the results from the cell lines, miR-342-3p expression was significantly decreased in OSCC tumor tissues compared with that in the adjacent non-cancerous specimens (Fig. 1B). Together, the present data demonstrated that miR-342-3p expression was significantly downregulated in OSCC cells and tissues, suggesting that miR-342-3p may serve a role in the development of OSCC. Given that Tca8113 and SCC-9 cell lines express an intermediate level of miR-342-3p compared with other OSCC cell lines (OC3, SCC-4 and OEC-M1) (Fig. 1A), they were selected for subsequent gain-of-function and loss-of-function experiments.

Upregulation of $\mathrm{miR}-342-3 p$ inhibits the proliferation of OSCC cells. The reduced expression of miR-342-3p in OSCC samples suggested that it may function as a tumor suppressor. To investigate the role of miR-342-3p, miR mimics were transfected into the Tca8113 and SCC-9 cells. As shown in Fig. 2A and B, the expression of miR-342-3p in the Tca8113 and SCC-9 cells was significantly increased following transfection with an miR-342-3p-mimic. CCK-8 assay results showed that ectopic expression of miR-342-3p significantly inhibited the proliferation of the Tca8113 and SCC-9 cells (Fig. 2C and D). In addition, miR-342-3p overexpression resulted in a marked decrease in the clonogenic potential of the Tca8113 and SCC-9 cells (Fig. 2E and F). In accordance with the results from the CCK-8 assay, the BrdU-incorporation assay revealed the suppression of OSCC cell proliferation by miR-342-3p mimic transfection (Fig. 2G and $\mathrm{H}$ ). Taken together, these findings suggest that miR-342-3p inhibits the proliferation of OSCC cells.

Inhibition of $m i R-342-3 p$ promotes the proliferation of OSCC cells. To confirm the suppressive role of miR-342-3p in the proliferation of OSCC cells, the effect of miR-342-3p inhibition on OSCC cell proliferation was explored. AntagomiR-342-3p was transfected into the Tca8113 and SCC-9 cells to silence the expression of endogenous miR-342-3p. As shown in Fig. 3A and B, endogenous miR-342-3p expression was significantly downregulated in the Tca8113 and SCC-9 cells following antagomiR-342-3p transfection. CCK-8 assays revealed that the inhibition of miR-342-3p markedly promoted the proliferation of the Tca8113 and SCC-9 cells (Fig. 3C and D). Furthermore, the colony-forming ability of the Tca8113 and SCC-9 cells was notably enhanced by antagomiR-342-3p transfection (Fig. 3E and F). The BrdU-incorporation assay further demonstrated that the Tca 8113 and SCC-9 cells transfected with antagomiR-342-3p exhibited a marked increase in cell proliferation ability compared with the cells transfected with antagomiR negative control (Fig. $3 \mathrm{G}$ and $\mathrm{H}$ ). Overall, these results indicate that the inhibition of miR-342-3p promotes the proliferation of OSCC cells.

miR-342-3p directly targets LASP1 in OSCC cells. Notably, LASP1, an oncogene implicated in the initiation and progression of OSCC (22), was found to possess the putative binding sites for miR-342-3p in its 3'-UTR (Fig. 4A). To determine the direct binding between miR-342-3p and the 3'-UTR of LASP1, the WT LASP1 3'-UTR and a 3'-UTR with mutations in the predicted miR-342-3p binding site were cloned into a luciferase reporter plasmid. Luciferase reporter assays showed that miR-342-3p overexpression significantly suppressed the luciferase activity in the Tca8113 and SCC-9 cells transfected with the WT 3'-UTR plasmid, whereas the enforced expression miR-342-3p had little effect on the luciferase activity in the cells transfected with plasmid carrying the mutant binding site (Fig. 4B). Additionally, miR-342-3p inhibition notably promoted the luciferase activity in the Tca8113 and SCC-9 cells with the plasmid carrying the WT 3'-UTR, whereas the enhanced effect was substantially reverted when the miR-342-3p binding sites on the plasmid were mutated, suggesting that LASP1 is a direct target of miR-342-3p (Fig. 4C). Moreover, RT-qPCR and western blot analysis revealed that miR-342-3p overexpression significantly decreased the mRNA and protein expression of LASP1 in the Tca8113 and SCC-9 cells, while the inhibition of endogenous miR-342-3p markedly increased LASP1 expression at the mRNA and protein levels (Fig. 4D-G). Taken 

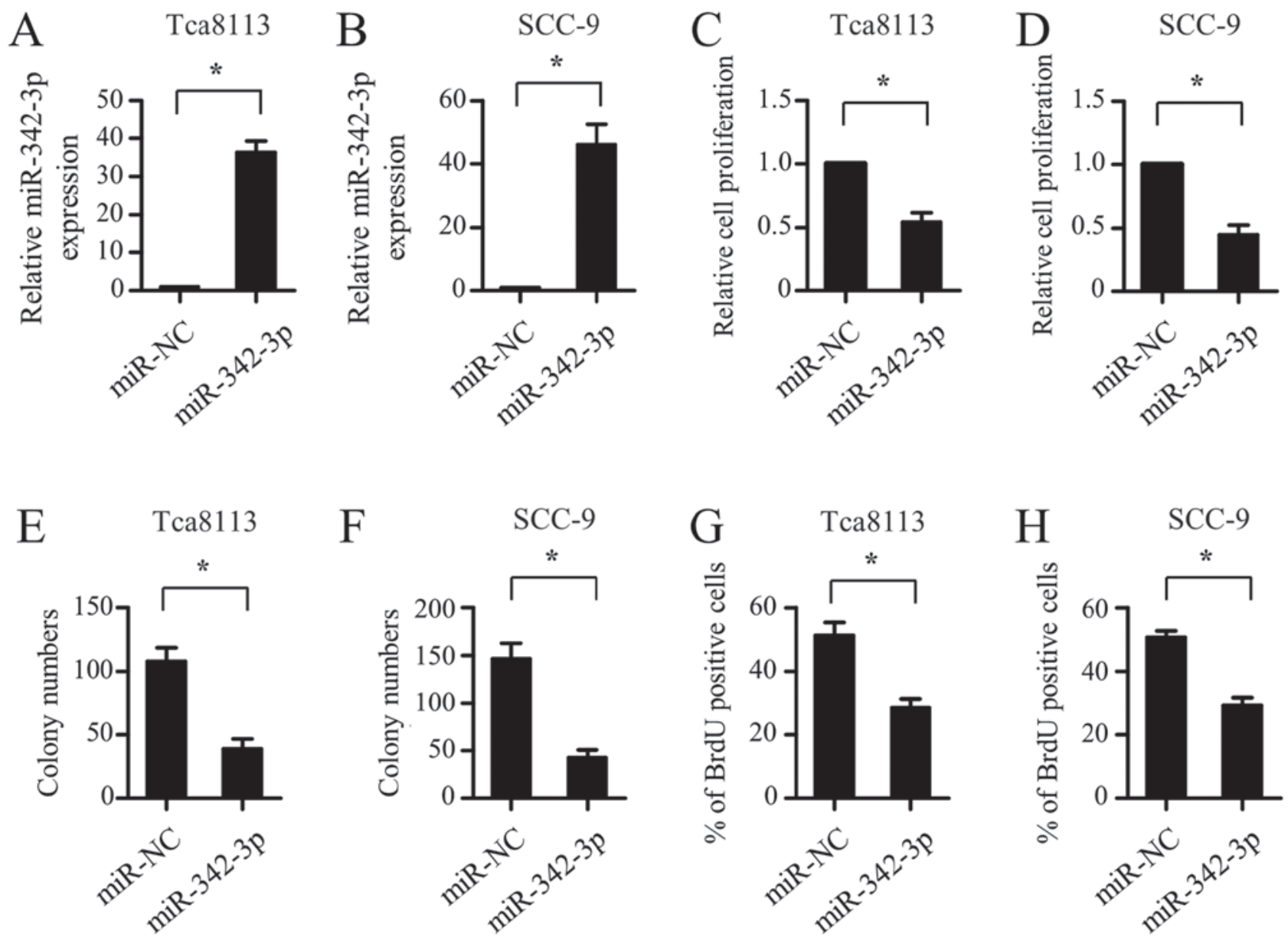

Figure 2. Upregulation of miR-342-3p inhibits the proliferation of oral squamous cell carcinoma cells. Quantitative PCR analysis of miR-342-3p expression in (A) Tca8113 and (B) SCC-9 cells transfected with miR-NC or miR-342-3p, normalized to the expression in non-transfected control cells of the same line. Tca8113 and SCC-9 cells were transfected with miR-NC or miR-342-3p. After $24 \mathrm{~h}$, the following tests were performed: The Cell Counting kit-8 assay to calculate the relative cell proliferation in (C) Tca8113 and (D) SCC-9 cells, normalized to the cell proliferation in control cells of the same line; the colony formation assay to give colony counts for (E) Tca8113 and (F) SCC-9 cells; and the BrdU-incorporation assay to give percentage positivity for (G) Tca8113 and (H) SCC-9 cells. All the assays were performed in triplicate and the data are shown as the mean \pm standard deviation. ${ }^{*} \mathrm{P}<0.05$. miR, microRNA; NC, negative control; BrdU, 5-bromo-2-deoxyuridine.

together, these data indicate that miR-342-3p negatively regulates the expression of LASP1 through directly targeting its 3'-UTR. The correlation between miR-342-3p and LASP1 levels in clinical specimens was also explored. A total of 10 pairs of tumor tissues and the adjacent non-cancerous specimens from the same patients were collected, and the expression of miR-342-3p and LASP1 mRNA was tested. As demonstrated in Fig. 4H, when the relative expression levels (OSCC/normal) of LASP1 were plotted against those of miR-342-3p for each patient, a significant inverse correlation was revealed $(\mathrm{P}<0.0053 ; \mathrm{R}=-0.8011)$. These data suggest that miR-342-3p downregulation is associated with the increase in LASP1 in OSCC tumor tissues.

miR-342-3p suppresses the proliferation of OSCC cells by inhibiting LASP1. To investigate whether miR-342-3p suppresses the cell proliferation of OSCC cells by targeting LASP1, the 3'-UTR-deleted LASP1 plasmid was co-transfected with miR-342-3p mimic into SCC-9 cells, and the cell proliferation ability was determined using CCK-8 and BrdU-incorporation assays. As shown in Fig. 5A-C, the miR-342-3p mimic transfection resulted in decreased cell proliferation of OSCC cells, whereas this inhibitory effect was completely reversed by LASP1 plasmid transfection. Furthermore, LASP1 silencing with siLASP1 markedly abrogated the SCC- 9 cell proliferation increase that was caused by miR-342-3p inhibition (Fig. 5D-F). These results support the hypothesis that miR-342-3p targets LASP1 to inhibit the proliferation of OSCC cells.

\section{Discussion}

Increasing evidence demonstrates that miRNA deregulation serves a key role in carcinogenesis. Therefore, miRNAs have been investigated extensively to identify novel diagnostic and prognostic cancer biomarkers, and to aid the development of effective therapeutic targets. In OSCC, various miRNAs are abnormally expressed and participate in cancer initiation and progression (23). For example, miR-155 was reported to exhibit a significant upregulation in OSCC tissues compared within their matched normal samples (24). Moreover, miR-155 promotes OSCC development by downregulating tumor suppressor protein cell division cycle 73 (25). By contrast, miR-9 shows decreased expression in OSCC tissues and 

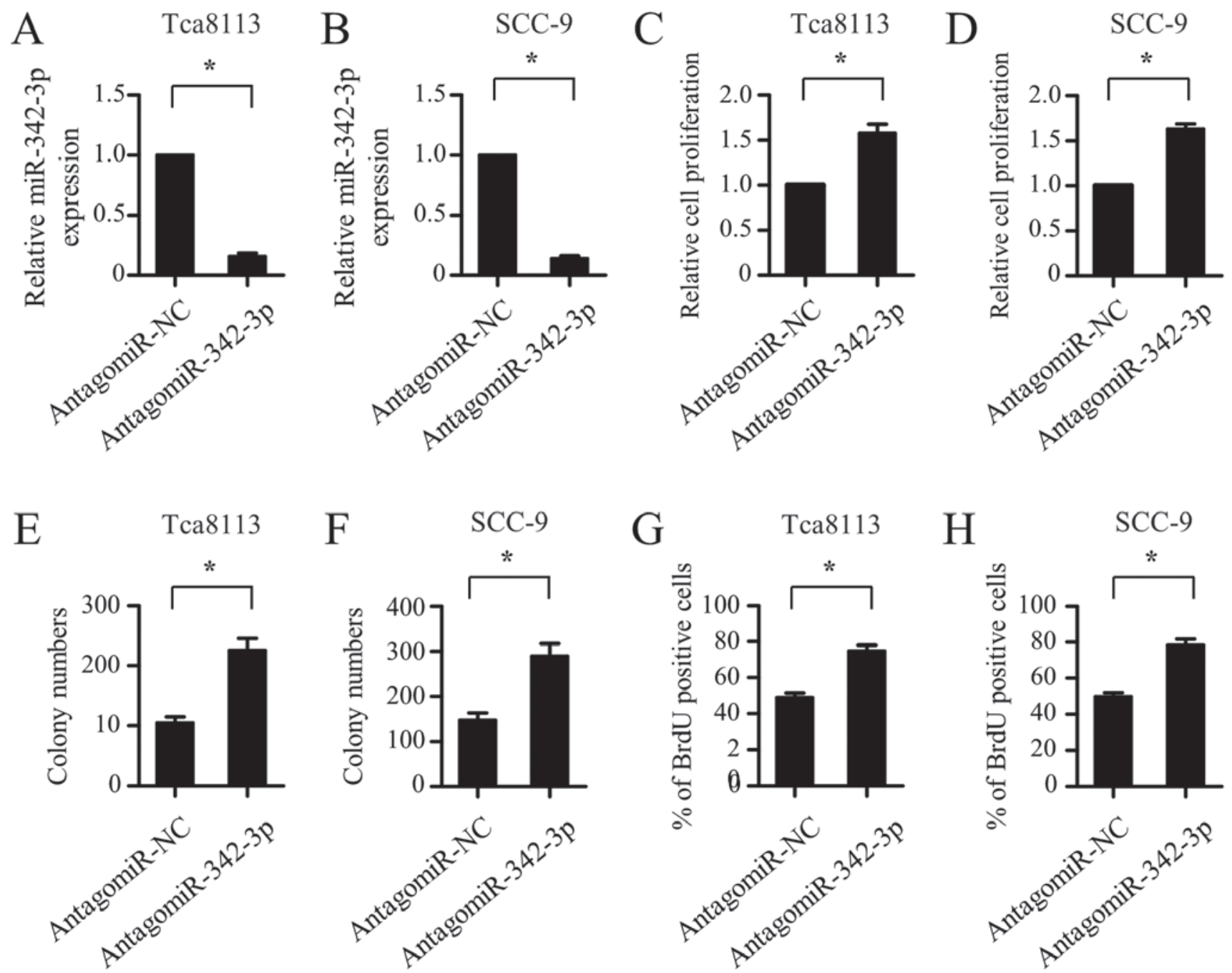

Figure 3. Inhibition of miR-342-3p promotes the growth of oral squamous cell carcinoma cells. Quantitative PCR analysis of miR-342-3p expression in (A) Tca8113 and (B) SCC-9 cells transfected with antagomiR-NC or antagomiR-342-3p, normalized to the level of miR-342-3p in non-transfected control cells of the same line. After $24 \mathrm{~h}$ of transection, the following tests were performed: The Cell Counting kit- 8 assay to calculate the relative cell proliferation in (C) Tca8113 and (D) SCC-9 cells, normalized to the cell proliferation in control cells of the same line; the colony formation assay to provide colony counts for (E) Tca8113 and (F) SCC-9 cells; and the BrdU-incorporation assay to provide percentage positivity for (G) Tca8113 and (H) SCC-9 cells. All data are shown as the mean \pm standard deviation of three separate experiments. " $\mathrm{P}<0.05$. miR, microRNA; NC, negative control; BrdU, 5-bromo-2-deoxyuridine.

serves a tumor-suppressor role by impairing the expression of miR-99a and inactivating Wnt/ $\beta$-catenin signaling (10).

Previous studies have reported the involvement of miR-342-3p in different malignancies, including hepatocellular carcinoma, cervical cancer and lung cancer (17-19,26-28). However, the role of miR-342-3p in OSCC remains unknown. The present study demonstrated that miR-342-3p exhibited decreased expression in OSCC tissues when compared with that in adjacent non-tumorous tissues, suggesting that miR-342-3p may serve a tumor-suppressing role. Further experiments confirmed the inhibitory role of miR-342-3p in the proliferation of OSCC cells, as assessed by the CCK-8, colony formation and BrdU-incorporation assays. Collectively, these tests indicate that miR-342-3p acts as a tumor suppressor in OSCC, which is consistent with its role in a number of tumors. Previous studies also revealed the crucial role of miR-342-3p in tumor metastasis $(18,19)$; however, whether it affects the cell migration of OSCC cells requires further investigation. It has been reported that miR-9, 99a and 491-5p are downregulated in OSCC and exhibit tumor-suppressive activities (10-13). The downregulation level of miR-342-3p was compared with those of miR-9, 99a and 491-5p in OSCC tissues relative to normal tissues and it was found to be analogous.

LASP1, a member of the LIM proteins, was initially identified from a cDNA library of human breast cancer tissues (29). LASP1 is ubiquitously expressed in all normal tissues and participates in a wide spectrum of cellular processes (30). A growing number of studies suggest that LASP1 is overexpressed in numerous malignant tumors and is correlated with poor clinical prognoses for patients with different cancer types, including ovarian, breast, bladder, NSCLC and colorectal cancer (31-35). LASP1 was reported to exhibit increased expression in patients with OSCC and was significantly correlated with the primary tumor size (22). Further study demonstrated that LASP1 promotes OSCC cell proliferation through accelerating cell-cycle progression (22). However, the mechanism underlying LASP1 regulation remains to be investigated. A growing body of evidence has revealed the critical role of miRNAs in 


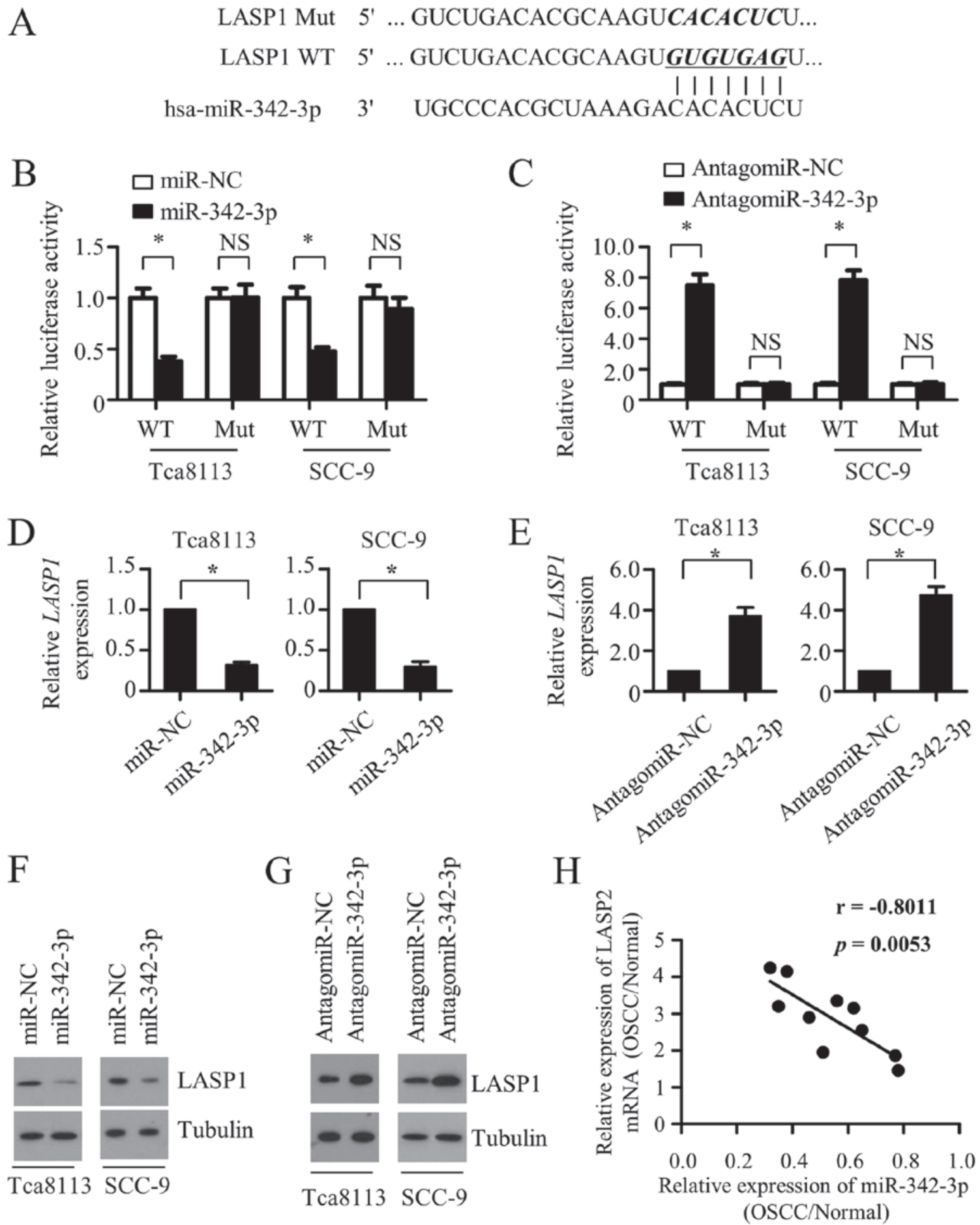

Figure 4. miR-342-3p directly targets LASP1 in OSCC cells. (A) Predicted miR-342-3p target sequence in the 3 -UTR of LASP1 mRNA, and the WT and mutated versions used in the luciferase reporter plasmids. Luciferase activity data (shown as the mean \pm SD of triplicate measurements) of (B) Tca8113 and SCC-9 cells transfected with WT or mutant LASP1 3'-UTR luciferase reporter plasmid along with miR-NC or miR-342-3p, and (C) Tca8113 and SCC-9 cells co-transfected with antagomiR-NC or antagomiR-342-3p and WT or mutant LASP1 3'-UTR luciferase reporter vectors. Quantitative PCR was performed to detect the expression of LASP1 mRNA in Tca8113 and SCC-9 cells transfected with (D) miR-NC or miR-342-3p or (E) antagomiR-NC or antagomiR-342-3p. Expression of LASP1 mRNA was normalized to GAPDH. Results are shown as the mean \pm SD of triplicate measurements. (F) Western blotting was used to measure the expression of LASP1 protein in Tca8113 and SCC-9 cells transfected with (F) miR-NC or miR-342-3p or (G) antagomiR-NC or antagomiR-342-3p $\alpha$-tubulin was used as a loading control. (H) A statistically significant inverse correlation between miR-342-3p expression and LASP1 mRNA in OSCC vs. normal tissues from the same patient was observed following Pearson's correlation analysis $(\mathrm{R}=-0.8011 ; \mathrm{P}=0.0053)$. "P<0.05. miR, microRNA; LASP1, LIM and SH3 protein 1; OSCC, oral squamous cell carcinoma; 3'-UTR, 3' untranslated region; WT, wild-type; NC, negative control; SD, standard deviation; NS, not significant.

controlling LASP1 expression in an increasing number of cancer types. For example, miR-203a-3p suppressed cell proliferation and migration by directly targeting LASP1 in nasopharyngeal carcinoma (36), and miR-203 was reported to inhibit tumor proliferation by repressing LASP1 expression in NSCLC (34). In the present study, LASP1 was identified as a potential target 


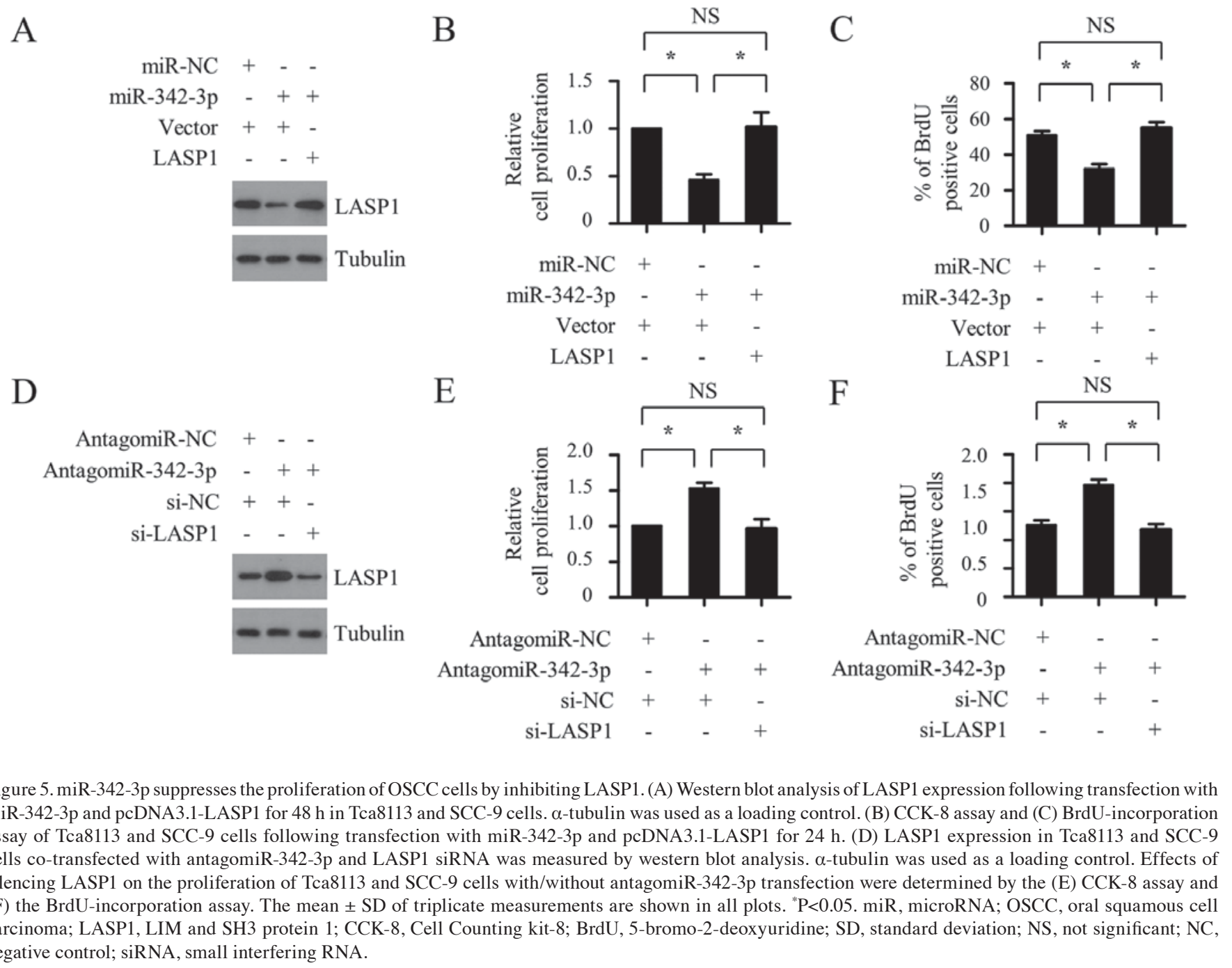

gene of miR-342-3p through bioinformatic prediction analysis. A luciferase reporter assay revealed that miR-342-3p inhibited the expression of LASP1 by directly binding to its 3'-UTR. RT-qPCR and western blot analysis confirmed the suppressive role of miR-342-3p in LASP1 expression. Furthermore, the present results demonstrated that LASP1 overexpression effectively reversed the suppressive effect of miR-342-3p on OSCC cell proliferation, validating the functional significance of LASP1 in mediating the tumor suppressor role of miR-342-3p. Although these studies demonstrate that miR-342-3p inhibits OSCC cell proliferation by directly targeting LASP1, the correlation between miR-342-3p and LASP1 expression in clinical OSCC tissues remains to be investigated.

In summary, the present study demonstrated that miR-342-3p functions as a tumor suppressor through downregulation of LASP1 expression in OSCC. Novel insights into molecular mechanisms regulating OSCC progression have been provided, as well as a promising molecular target for the development of a novel, more efficacious therapeutic approach for the treatment of patients with OSCC.

\section{Acknowledgements}

Not applicable.

\section{Funding}

The present study was supported by the Research Program of Science and Technology of Baotou (grant no. wsjj2011037).

\section{Availability of data and materials}

The datasets used and/or analyzed during the current study are available from the corresponding author on reasonable request.

\section{Authors' contributions}

$\mathrm{BC}$ coordinated and designed the project, designed the experiments and edited the manuscript. XS, YJ, MY and YZ performed the experiments. XS and YJ analyzed the data and wrote the manuscript.

\section{Ethics approval and consent to participate}

The present study was approved by the Ethics Committee of the Third Affiliated Hospital, Inner Mongolia Medical University (Baotou, Inner Mongolia, China). All participants provided written informed consent. 


\section{Patient consent for publication}

Written informed consent for publication was obtained from the patient.

\section{Competing interests}

The authors declare that they have no competing interests.

\section{References}

1. Scully C and Bagan J: Oral squamous cell carcinoma: Overview of current understanding of aetiopathogenesis and clinical implications. Oral Dis 15: 388-399, 2009.

2. Ishida K, Tomita H, Nakashima T, Hirata A, Tanaka T, Shibata T and Hara A: Current mouse models of oral squamous cell carcinoma: Genetic and chemically induced models. Oral Oncol 73 16-20, 2017.

3. Gharat SA, Momin M and Bhavsar C: Oral squamous cell carcinoma: Current treatment strategies and nanotechnology-based approaches for prevention and therapy. Crit Rev Ther Drug Carrier Syst 33: 363-400, 2016.

4. Rupaimoole R, Calin GA, Lopez-Berestein G and Sood AK miRNA deregulation in cancer cells and the tumor microenvironment. Cancer Discov 6: 235-246, 2016.

5. Rupaimoole R and Slack FJ: MicroRNA therapeutics: Towards a new era for the management of cancer and other diseases. Nat Rev Drug Discov 16: 203-222, 2017.

6. Ling H, Fabbri $M$ and Calin GA: MicroRNAs and other non-coding RNAs as targets for anticancer drug development Nat Rev Drug Discov 12: 847-865, 2013.

7. Reis PP, Tomenson M, Cervigne NK, Machado J, Jurisica I, Pintilie M, Sukhai MA, Perez-Ordonez B, Grénman R, Gilbert RW, et al: Programmed cell death 4 loss increases tumor cell invasion and is regulated by miR-21 in oral squamous cell carcinoma. Mol Cancer 9: 238, 2010.

8. Jiang F, Zhao W, Zhou L, Zhang L, Liu Z and Yu D: miR-222 regulates the cell biological behavior of oral squamous cell carcinoma by targeting PUMA. Oncol Rep 31: 1255-1262, 2014.

9. Shen L, Liu L, Ge L, Xie L, Liu S, Sang L, Zhan T and Li H: miR-448 downregulates MPPED2 to promote cancer proliferation and inhibit apoptosis in oral squamous cell carcinoma. Exp Ther Med 12: 2747-2752, 2016.

10. Yu T, Liu K, Wu Y, Fan J, Chen J, Li C, Yang Q and Wang Z: MicroRNA-9 inhibits the proliferation of oral squamous cell carcinoma cells by suppressing expression of CXCR4 via the Wnt/ $\beta$-catenin signaling pathway. Oncogene 33: 5017-5027, 2014

11. Yan B, Fu Q, Lai L, Tao X, Fei Y, Shen J, Chen Z and Wang Q: Downregulation of microRNA 99a in oral squamous cell carcinomas contributes to the growth and survival of oral cancer cells. Mol Med Rep 6: 675-681, 2012.

12. Yen YC, Shiah SG, Chu HC, Hsu YM, Hsiao JR, Chang JY, Hung WC, Liao CT, Cheng AJ, Lu YC and Chen YW: Reciprocal regulation of microRNA-99a and insulin-like growth factor I receptor signaling in oral squamous cell carcinoma cells. Mol Cancer 13: 6, 2014.

13. Huang WC, Chan SH, Jang TH, Chang JW, Ko YC, Yen TC, Chiang SL, Chiang WF, Shieh TY, Liao CT, et al: miRNA-491-5p and GIT1 serve as modulators and biomarkers for oral squamous cell carcinoma invasion and metastasis. Cancer Res 74: 751-764, 2014.

14. Czimmerer Z, Varga T, Kiss M, Vázquez CO, Doan-Xuan QM, Rückerl D, Tattikota SG, Yan X, Nagy ZS, Daniel B, et al: The IL-4/STAT6 signaling axis establishes a conserved microRNA signature in human and mouse macrophages regulating cell survival via miR-342-3p. Genome Med 8: 63, 2016.

15. Wang L, Xu L, Xu M, Liu G, Xing J, Sun C and Ding H: Obesity-associated MiR-342-3p promotes adipogenesis of mesenchymal stem cells by suppressing CtBP 2 and releasing $\mathrm{C} / \mathrm{EBP} \alpha$ from CtBP2 binding. Cell Physiol Biochem 35: 2285-2298, 2015.

16. Huang M, Qing Y, Shi Q, Cao Y and Song K: miR-342-3p elevates osteogenic differentiation of umbilical cord mesenchymal stem cells via inhibiting Sufu in vitro. Biochem Biophys Res Commun 491: 571-577, 2017.
17. Gao Y, Zhang SG, Wang ZH and Liao JC: Down-regulation of miR-342-3p in hepatocellular carcinoma tissues and its prognostic significance. Eur Rev Med Pharmacol Sci 21: 2098-2102, 2017.

18. Xue X, Fei X, Hou W, Zhang Y, Liu L and Hu R: miR-342-3p suppresses cell proliferation and migration by targeting AGR2 in non-small cell lung cancer. Cancer Lett 412: 170-178, 2018.

19. Li XR, Chu HJ, Lv T, Wang L, Kong SF and Dai SZ: miR-342-3p suppresses proliferation, migration and invasion by targeting FOXM1 in human cervical cancer. FEBS Lett 588: 3298-3307, 2014.

20. Livak KJ and Schmittgen TD: Analysis of relative gene expression data using real-time quantitative PCR and the 2(-Delta Delta C(T)) method. Methods 25: 402-408, 2001.

21. Roberts KP, Ensrud KM and Hamilton DW: A comparative analysis of expression and processing of the rat epididymal fluid and sperm-bound forms of proteins D and E. Biol Reprod 67: 525-533, 2002.

22. Shimizu F, Shiiba M, Ogawara K, Kimura R, Minakawa Y, Baba T, Yokota S, Nakashima D, Higo M, Kasamatsu A, et al: Overexpression of LIM and SH3 Protein 1 leading to accelerated G2/M phase transition contributes to enhanced tumourigenesis in oral cancer. PLoS One 8: e83187, 2013.

23. Min A, Zhu C, Peng S, Rajthala S, Costea DE and Sapkota D: MicroRNAs as important players and biomarkers in oral carcinogenesis. Biomed Res Int 2015: 186904, 2015.

24. Shi LJ, Zhang CY, Zhou ZT, Ma JY, Liu Y, Bao ZX and Jiang WW: MicroRNA-155 in oral squamous cell carcinoma: Overexpression, localization, and prognostic potential. Head Neck 37: 970-976, 2015.

25. Rather MI, Nagashri MN, Swamy SS, Gopinath KS and Kumar A: Oncogenic microRNA-155 down-regulates tumor suppressor CDC73 and promotes oral squamous cell carcinoma cell proliferation: Implications for cancer therapeutics. J Biol Chem 288: 608-618, 2013.

26. Zhao L and Zhang Y: miR-342-3p affects hepatocellular carcinoma cell proliferation via regulating NF- $\mathrm{KB}$ pathway. Biochem Biophys Res Commun 457: 370-377, 2015.

27. Xie X, Liu H, Wang M, Ding F, Xiao H, Hu F, Hu R and Mei J: miR-342-3p targets RAP2B to suppress proliferation and invasion of non-small cell lung cancer cells. Tumour Biol 36: 5031-5038, 2015

28. Tai MC, Kajino T, Nakatochi M, Arima C, Shimada Y, Suzuki M, Miyoshi H, Yatabe Y, Yanagisawa K and Takahashi T: miR-342-3p regulates MYC transcriptional activity via direct repression of E2F1 in human lung cancer. Carcinogenesis 36: 1464-1473, 2015

29. Tomasetto C, Moog-Lutz C, Régnier CH, Schreiber V, Basset $P$ and Rio MC: Lasp-1 (MLN 50) defines a new LIM protein subfamily characterized by the association of LIM and SH3 domains. FEBS Lett 373: 245-249, 1995.

30. Orth MF, Cazes A, Butt E and Grunewald TG: An update on the LIM and SH3 domain protein 1 (LASP1): A versatile structural, signaling, and biomarker protein. Oncotarget 6: 26-42, 2015.

31. Grunewald TG, Kammerer U, Winkler C, SchindlerD, Sickmann A, Honig A and Butt E: Overexpression of LASP-1 mediates migration and proliferation of human ovarian cancer cells and influences zyxin localisation. Br J Cancer 96: 296-305, 2007.

32. Grunewald TG, Kammerer U, Schulze E, Schindler D, Honig A, Zimmer M and Butt E: Silencing of LASP-1 influences zyxin localization, inhibits proliferation and reduces migration in breast cancer cells. Exp Cell Res 312: 974-982, 2006.

33. Chiyomaru T, Enokida H, Kawakami K, Tatarano S, Uchida Y, Kawahara K, Nishiyama K, Seki N and Nakagawa M: Functional role of LASP1 in cell viability and its regulation by microRNAs in bladder cancer. Urol Oncol 30: 434-443, 2012.

34. Zheng J, Wang F, Lu S and Wang X: LASP-1, regulated by miR-203, promotes tumor proliferation and aggressiveness in human non-small cell lung cancer. Exp Mol Pathol 100: 116-124, 2016.

35. Zhao L, Wang H, Liu C, Liu Y, Wang X, Wang S, Sun X, Li J, Deng Y, Jiang Y and Ding Y: Promotion of colorectal cancer growth and metastasis by the LIM and SH3 domain protein 1 . Gut 59: 1226-1235, 2010.

36. Jiang N, Jiang X, Chen Z, Song X, Wu L, Zong D, Song D, Yin L, Wang D, Chen C, et al: MiR-203a-3p suppresses cell proliferation and metastasis through inhibiting LASP1 in nasopharyngeal carcinoma. J Exp Clin Cancer Res 36: 138, 2017. 